\title{
Predictors and Direct Cost Estimation of Long Stays in Pediatric Intensive Care Units in Saudi Arabia: A Mixed Methods Study
}

\author{
Mohamad- \\ Hani Temsah (D) ${ }^{1,2, *}$ \\ Noura Abouammoh ${ }^{2,3, *}$ \\ Ayman Al-Eyadhy $\mathbb{D}^{1,2}$ \\ Yazed AIRuthia (iD) 4,5 \\ Marwah Hassounah (D) ${ }^{3}$ \\ Fahad Alsohime ${ }^{1,2}$ \\ Ali Alhaboob ${ }^{1,2}$ \\ Mohammed Almazyad (ID) ${ }^{1,2}$ \\ Majed Alabdulhafid ${ }^{1,2}$ \\ Fadi Aljamaan $\mathbb{D D}^{2,6}$ \\ Khalid Alhasan $\mathbb{D}^{1,2}$ \\ Amr Jamal (D) $2,3,7$
}

\begin{abstract}
'Department of Pediatrics, College of Medicine, King Saud University, Riyadh, Saudi Arabia; ${ }^{2}$ King Saud University Medical City, King Saud University, Riyadh, Saudi Arabia; ${ }^{3}$ Family and Community Medicine Department, College of Medicine, King Saud University, Riyadh, Saudi Arabia; ${ }^{4}$ Department of Clinical Pharmacy, College of Pharmacy, King Saud University, Riyadh, Saudi Arabia; ${ }^{5}$ Pharmacoeconomics Research Unit, College of Pharmacy, King Saud University, Riyadh, Saudi Arabia; ${ }^{6}$ Department of Critical Care, College of Medicine, King Saud University, Riyadh, Saudi Arabia; ${ }^{7}$ Evidence-Based Health Care \& Knowledge Translation Research Chair, King Saud University, Riyadh, Saudi Arabia
\end{abstract}

*These authors contributed equally to this work

Correspondence: Amr Jamal

Email amrjamal@ksu.edu.sa
Background: Several factors influence patients' length of stay (LOS) in pediatric intensive care units (PICUs). This study explored the factors and cost associated with long LOS among pediatric patient population across various PICUs and pediatric surgical cardiac intensive care units (SCICUs).

Methods: This is a multi-center concurrent mixed-methods study that was conducted in different PICUs and SCICUs in Riyadh, Saudi Arabia. The units' leaders were qualitatively interviewed to explore the potential factors contributing to long LOSs in PICUs and SCICUs, and deductive content analysis was used. Microcosting was utilized to estimate the inpatient costs for a sample of long-stay patients (LSPs) and the potential cost savings if those patients were transferred to step-down care units (SDUs) for those who stayed $\geq 60$ days.

Results: Ten (90\%) leaders of PICUs and SCICUs who were invited had participated. Changing the admission criteria, patient transfer to SDUs, family engagement in the decision-making process, and adopting a national do-not-resuscitate (DNR) policy were factors contributing to short LOSs. On the other hand, administrative constraints, staff avoidance of code status decisions, lack of palliative care resources, and complex patient characteristics were factors associated with long LOSs. The mean inpatient cost per patient was SAR 3.63 million (USD 0.97 million), and the total cost for the 48 patients was SAR 172.95 million (USD 46.12 million).

Conclusion: If the recommendation to transfer LSPs after a 60-day PICU stay to SDUs is taken, the estimated cost savings among this sample of patients alone can be as high as SAR 109.47 million (USD 29.19 million). Therefore, future studies should examine the factors contributing to long LOSs in PICUs and SCICUs as well as the effectiveness of different policies aimed at improving the resource allocation and management using more robust study designs and diverse patient population.

Keywords: pediatric critical care, long-stay patients, cost of long-stay patients, content analysis

\section{Introduction}

Intensive care units (ICU) were invented to resuscitate and manage a patient while passing through a critical illness. Providing prompt access to acute care beds requires effective and efficient utilization of ICU beds. A long-stay patient (LSP) in an ICU is defined as a patient who survived an acute critical illness but still requires intense monitoring or support. As such, patients require prolonged intensive care admission beyond the average length of stay expected at their initial 
admittance for their diagnosis and disease severity; more resources and financial costs will be needed for their hospitalizations. The definition of a long stay in pediatric intensive care units (PICUs) differs in the literature, ranging from 13 to 56 days. ${ }^{1-6}$ Others have suggested using a definition of a length of stay above the 95th percentile of the ICU population length of stay, proposing a definition of LSP as being an ICU stay that extends beyond 14 days. $^{7}$

While LSPs might represent a minority of total ICU bed occupancy, they need a disproportionately large amount of resources. ${ }^{3}$ Furthermore, these patients occupy the already limited number of ICU beds. ${ }^{5}$ Different approaches have been attempted to improve resource utilization in PICUs; however, they have been challenged by the unavoidable presence of LSPs. ${ }^{1}$ There are inconsistencies in the published data regarding pediatric LSP prevalence, with limited data regarding some regions with rapidly evolving pediatric critical care services. Exploring this issue might be more pertinent in countries with a growing population, such as Saudi Arabia, where children represent $37.2 \%$ of the population compared to $20 \%$ in the United States (US). ${ }^{8}$ Locally, the issue of LSP was showing a trend of decrease, with the prevalence of LSP in several hospitals in Saudi Arabia decreasing from $32 \%$ in 2014 to $23.4 \%$ in $2019 .{ }^{9}$ Nevertheless, the impact of LSP in PICUs and factors related to this still need to be addressed.

This study aimed to explain our previous quantitative findings on the change in LSP prevalence and characteristics $^{9}$ in Saudi Arabia and provide practical and relevant recommendations to decision-makers. For that, we obtained insights from multiple PICU and pediatric surgical cardiac intensive care unit (SCICU) leaders about the perceived factors that improved or worsened LSP outcomes in their settings over the past five years. Also, we estimated the current operational cost of hospitalization for these LSP versus the cost with a proposed alternate model that recommends the transfer of LSP patients with a length of stay of $\geq 60$ days to a step-down unit (SDU)

\section{Methods}

This paper is a continuum to previous research by the authors, ${ }^{9}$ which explains data source, data collection tool, recruitment, and data collection process. The same seven PICUs and three pediatric SCICUs in Riyadh, Saudi Arabia, were included in the current study. This paper seeks to understand further the quantitative results we previously found using qualitative methodology and estimate possible financial gain by reducing LSPs using microcosting method. [Figure 1]

Qualitative analysis was used to provide in-depth information regarding the perceived reasons for long stays among LSPs in ICUs. Data were collected using narrative technique. Questions were sent electronically to the PICU heads, or their delegates. All participants provided their insights within three weeks of starting the data collection process (26 Aug-15 Sep 2019). Follow-up reminders were sent to non-respondents by WhatsApp messages and phone calls.

Data collection for the current study included four open-ended questions: what were the main reasons of change in their Unit's number of LSPs, how did they manage to address the issues of having LSP during the previous 5 years, what issues positively impacted their LSP, and what issues negatively impacted their LSP. Emphasis was given in each question to inform the research team as much as possible and to send their voice message for more details, if needed. These questions were piloted among two PICU consultants, with minor changes in the question phrasing, to elicit more opinions from the participants, while ensuring clarity and consistency.

Deductive directed content analysis was then performed. Data for content analysis consisted of three phases: the preparatory phase, organization phase, and reporting phase. ${ }^{10}$ The preparatory phase included collecting data from the ICUs' heads. The unit of analysis was decided to be any expression that referred to factors negatively or positively affecting ICU LSPs. All responses were in text, except for one that in audio format. The audio response was transcribed by the primary author to text. Within each response, there was a mix of English and Arabic language and the research team is bilingual with professional level proficiency of English. Very specific intensive care terminology new to the qualitative research analyst was clarified by the primary author who is an intensive care consultant.

The organization phase commenced after familiarization with the range of the responses. This included open coding of all responses, the creation of categories, and, finally, the creation of concepts. Two qualitative researchers conducted the organization phase. The last phase was the reporting phase, which comprised coming to an agreement on a conceptual category and presenting the results using narrative and quotations to enhance confirmability. 
1- The change in pediatric

LSPS prevalence and baseline

characteristics in Riyadh,

Saudi Arabia (2014-2019)

[quantitative analysis]
2.1-Unit leaders' narrative

insights to factors positively

or negatively affecting the change in prevalence

[qualitative analysis]
2.2-Financial estimation of pediatric LSPs' and possible financial savings

if a proposed alternative is implemented

[quantitative analysis]

\section{3- To present rounded multi-perspective solutions and recommendations to decision makers regarding pediatric LSPs \\ [possible formats of presenting: report, presentation, in- person meeting]}

Figure I A diagram showing the pediatric intensive care units LSP research project scope.

For methodological rigor, this study involved multiple disciplines in the research team. The disciplines included multi-center pediatric intensive care providers, family medicine, and public health workers who had worked in the Saudi healthcare system. The pediatric intensivists had vast experience with the health problems addressed and were acquainted with the hospital unit heads' demanding schedules, language, and work culture. To accommodate the unit heads' demanding schedule, WhatsApp was chosen to survey the participants electronically and provide voice messages as an option for responses. The data were recorded via text responses to the electronic survey
(Survey Monkey platform, Supplementary Material) or through a recorded voice message. The analysis was iterative, with frequent visits to the raw data, allowing the analyst to practice reflexivity. The analyst $\mathrm{MH}$ discussed the categories and subcategories with the primary investigator (MHT) for contextual reference from a subjectmatter expert perspective, and NA revised the qualitative analysis methodology.

Moving on to the concurrent quantitative analysis, to estimate the cost of hospitalization for pediatric patients in PICUs, a review of the medical charts of hospitalized pediatric patients in 10 tertiary care hospitals in Riyadh, Saudi 


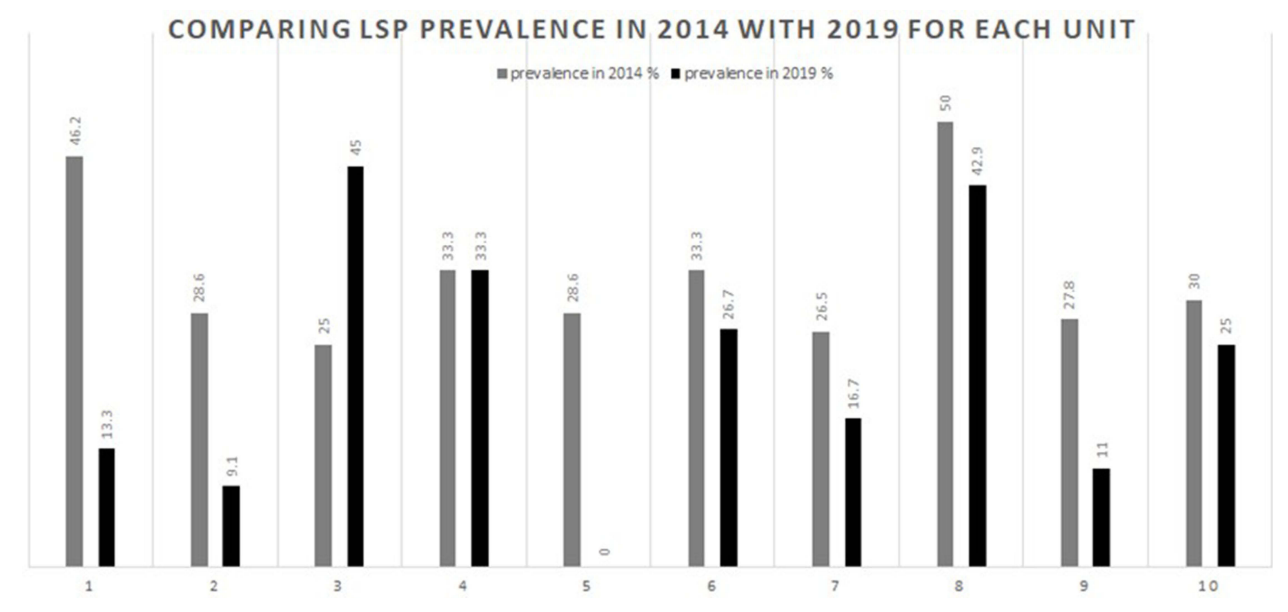

Figure 2 Comparing the LSP percentages for each PICU/SCICU, 2014 and 2019. Note: SCICUs are 2, 3, and 6; $\mathrm{p}$ value $<0.05$.

Arabia, hospitalized for more than 21 days in 2014 was conducted. The baseline data retrieved in September 2014 included the length of stay in PICUs, age at admission, the reason for admission, gender, and all inpatient services (eg, use of a ventilator, laboratory, and imaging studies, antibiotics use, use of inotropes, ICU or general ward room fee, etc.). The micro-costing method was applied to capture all inpatient services provided to patients during their hospitalization, such as the daily cost of mechanical ventilator use, medical consultation, prescription medication, and others. The costs of all provided inpatient services were retrieved from the cost center of the Saudi Ministry of Health. Descriptive statistics (eg, frequency, percentage, mean, and standard deviation, median with 25 th and 75 th percentile) were used to compare and contrast the cost per patient for the current policy with the new proposed policy of transferring patients who stayed over 60 days from the PICUs to a stepdown unit (SDUs). All statistical analyses were conducted using $\mathrm{SAS}^{\circledR}$ version 9.4 (SAS $^{\circledR}$ Institute, Cary, NC, US).

Approval from the Institutional Review Board at King Saud University was obtained before conducting the study (\#13/3793/IRB). The participating hospitals' names were coded to maintain confidentiality.

\section{Results}

\section{Descriptive Analysis results}

As reported in our previous publication, between 2014 and 2019, the LSP's overall percentage in all surveyed hospitals dropped by $7.5 \%$, from $31 \%$ to $23.5 \%$. ${ }^{9}$ At the hospital level, each unit varied in its improvement, from between a $5 \%$ to a 33\% drop in their LSP percentage (see Figure 2). One outlier unit had entirely resolved its LSP issue ( 0 cases in 2019 , from two cases and a percentage of $28.6 \%$ in 2014). On the other hand, one SCICU had a $20 \%$ increase in its LSP cases.

\section{Qualitative Analysis Results}

Directed content analysis of the textual responses provided the identification of the factors enabling and hindering the aforementioned individual variability in LSP percentage improvement. Under each category, several subcategories emerged, as shown in Figure 3. The participants' unit leaders consisted of eight males and two females (three delegates) from 10 PICUs/ SCICUs. Eight of the ten had held their position for over five years. Also, eight of the ten were the same unit heads that were interviewed for the 2014 survey. The following explains the categories and subcategories that emerged.

\section{Category I: Enabling Factors}

This category presents all of the factors proposed by the participants that contributed or might have contributed to decreasing the percentage of LSPs in PICUs and SCICUs. Five subcategories emerged from this category (see Figure 3 ).

\section{Acceptance and Transfer of Patients}

The participants saw that controlling the patient acceptance criteria and avoiding complex cases enabled them to decrease the likelihood of LSPs. Along with controlling the patient's entry into the PICU, the unit chairpersons believed that it was essential to have a firm policy regarding transferring a patient outside the unit when deemed medically reasonable before becoming an LSP. One SCICU leader communicated some ways to facilitate decreasing the number of LSPs: 


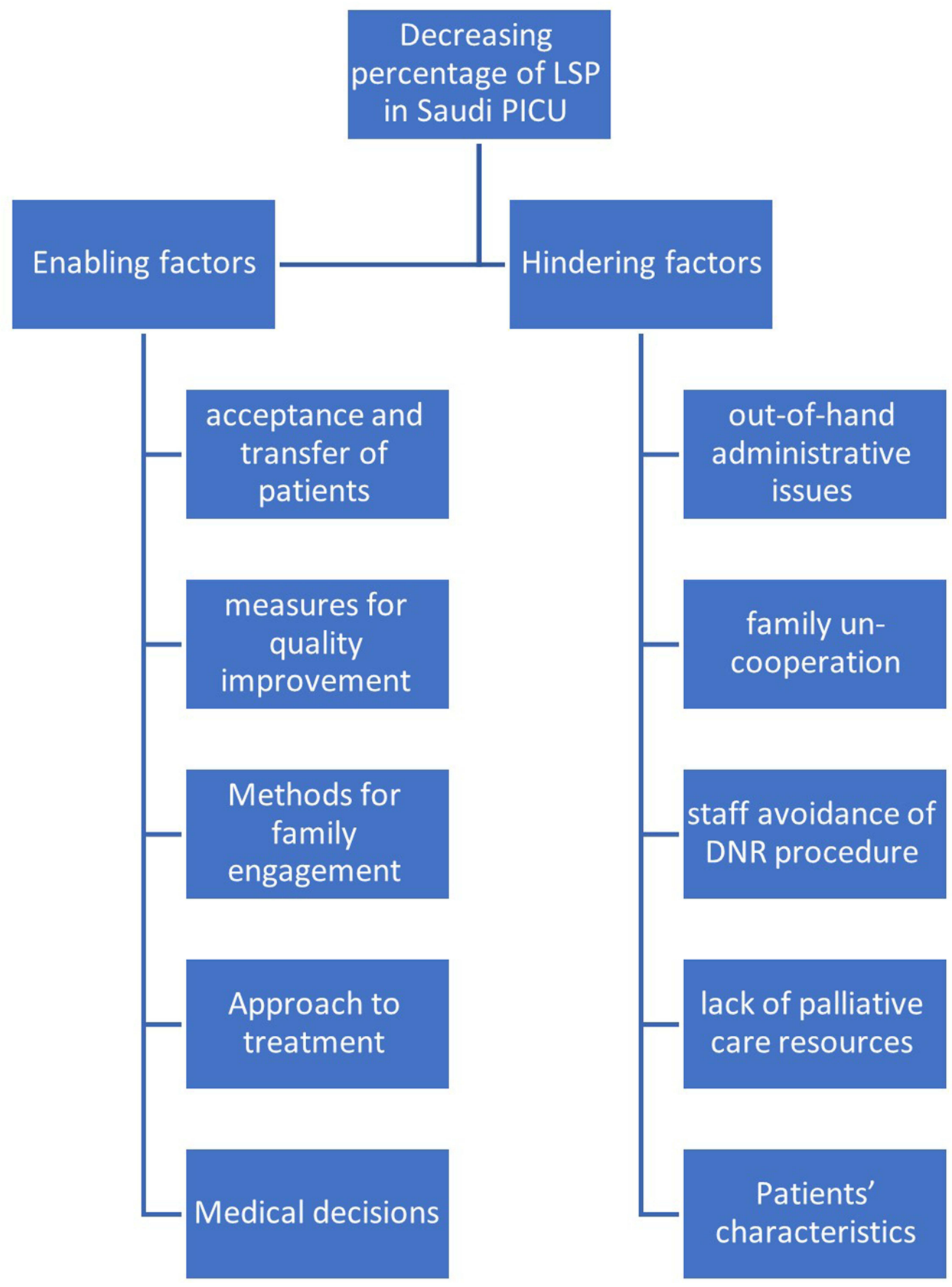

Figure 3 The emerging subcategories of the factors affecting the long-stay patients' percentage in PICUs.

"Address complex cases from all aspects: to better utilize health care system resources while aiming for good results and family satisfaction." -Hospital Unit \#6

Another participant recommended:

Suggest making an exchange system by which the chronic patients are sent to less busy units and hospitals, and accept referrals of acute cases from secondary hospitals to tertiary hospitals.
-Hospital Unit \#4

\section{Measures for Quality Improvement}

The participants discussed measures taken to improve their policies and procedures concerning LSPs. This included assigning a case or bed manager, having fast-tracked beds, having a clear guideline for the LSP pathway, complying with institutional accreditation requirements regarding palliative 
care, having a do-not-resuscitate (DNR) policy, and involving the quality department. A participant from a PICU commented:

"We started to practice KPIs [Key Performance Indicators], eg, length of hospital stays." - Hospital Unit \#4

They also noted:

Introduction of institutional accreditation by JCI [Joint Commission International] and CBAHI [Saudi Central Board for Accreditation of Healthcare Institutions] helped to accept palliative care practices in the PICU.

-Hospital Unit \#4

\section{Methods for Family Engagement}

There was an emphasis on involving the family in understanding the situation and the options available to facilitate a smooth transition of patients to palliative care, especially in medically hopeless cases or cases causing suffering for both the family and the patient. Among those methods are providing family counseling, education, and support and providing diagnostic tests to justify to the family putting the patient on DNR status and palliative care. One unit leader explained:

"Whole genome and whole exome help in confirming the diagnosis and approaching the families for palliative care when the case is DNR ..." -Hospital Unit \#5

Another participant from the ICU with no LSPs at the time of data collection explained:

"Family awareness is becoming more noticeable that helped us in making decisions." -Hospital Unit \#6

\section{Approach to Treatment}

The units' heads described an enabling approach to treatment as one with weekly LSP team meetings, a multidisciplinary approach with clear inter-disciplinary communication, support of new treatment modalities and early extubation, and "dis-intensification." Regarding the management plan, a participant from a SCICU with a significant increase in LSPs commented:

The other positive thing that I observed also is more dedicated team that are looking after cardiac patient, I'm talking about the nurses, the physicians, the RTs [respiratory therapist], other specialty involved; that make things actually better in terms of managing the patient properly.

-Hospital Unit \#3

\section{Medical Decisions}

There are two major medical decisions regarding LSPs that unit heads usually face. The first is deciding the goal of care. Subsequently, the second is the possibility of DNR status decisions based on the identified goals of care. Deciding care goals early and having the newly stated national end-of-life regulations facilitate avoiding patients' becoming LSPs.

One participant noted:

"Having national end-of-life regulations may have affected some of us in making decisions ..." -Hospital Unit \#10

\section{Category 2: Hindering Factors}

Participants further commented on the factors challenging their desire to decrease LSPs (Figure 3). Another five subcategories emerged from this category.

\section{Out-of-Hand Administrative Issues}

The unit heads mentioned several administrative factors that hindered their efforts in decreasing LSPs. One subcategory was process-related factors such as slow transfer to other facilities caring for LSPs and difficulty referring back patients to their original referring centers.

One PICU leader said:

Sometimes we get support from the $\mathrm{MOH}$ [Ministry of Health] to transfer some cases to private centers. No other new modalities and we are still struggling with difficulties in discharging cases.

-Hospital Unit \#10

\section{Lack of Family Cooperation}

Unit heads interpret families' negative attitude, and misunderstanding of the team's decisions for the goal of care and DNR status is interpreted as a hindering factor. This negative attitude leads to increased complaints, stirs public opinion, creates resistance to signing DNR forms, and promotes refusal to transfer or care for the patient.

One PICU head explained:

"The media utilized by the public to distort the facts and increase the burden on hospitals when making tough transfers or end-of-life decision"-Hospital Unit \#10

\section{Staff Avoidance of DNR}

Unit leaders highlighted the conflict and difficulty that the PICU teams confront when deciding on DNR status for LSPs. Choosing a DNR status might prevent conversion to 
an LSP or at least facilitate the decisions regarding palliative care options and transfer out of the unit. However, some admitting or primary physicians have a negative attitude toward DNR decisions, which plays a role in their delay in signing DNR forms and therefore augments the LSP problem.

A PICU head explained the following as an example of the barriers to facilitating fast DNR decisions:

“... one neurologist consultant once mentioned in a DNR multidisciplinary case conference in the presence of the parents 'I will not help you kill the patient!!"” Hospital Unit \#1

\section{Lack of Palliative Care Resources}

Home healthcare services and long-term facilities are usually scarce. Specialized and multidisciplinary chronic care services, such as what some LSPs might need, are even more scarce, which is often a significant factor hindering those patients' discharge process or contributing to family reluctance and resistance to the discharge process. This was frequently identified as a hindering factor.

One participant explained:

Few hospitals accept complex cardiac patients; because they don't have a pediatric cardiologist or cardiac surgeon. Therefore, some healthcare settings are 'scared or even panicked' from treating such patients. So, our SCICU faced the problem of transferring them back. While we succeed in transfers for a few patients, but they are not really enough to free beds for new active cases. Therefore, we end up with patients staying with us for longer time periods, despite that the patients' primary cardiac abnormalities were fixed, so the patients stay in our SCICU just for nursing and general ICU care.

-Hospital Unit \#3

\section{Patient Characteristics}

The patient's pre-PICU morbidity and case complexity play a role in treatment, recovery, and expected stay length. This is related closely to creating wise and realistic acceptance criteria for the PICU in general, especially for such complex cases. The complexity here refers to syndromic cases and high-risk patients for pre- and posttreatment complications.

Participants noted:

“... Some complex cases in which no clear resolution of the problem was possible for a long time ..." -Hospital Unit \#10
The type of patient with very complex, high-risk, single ventricle, hypo-plastic left heart, with the strict criteria that we have now compared to before, they are still challenging and develop more complications after cardiac surgery ...

-Hospital Unit \#3

\section{Direct Cost Estimation}

The mean and median costs of hospitalization per patient and the total cost for the patient cohort are shown in Table 1. The mean cost of hospitalization for the total length of stay per patient is 3.63 million Saudi Riyals (SAR), equivalent to 0.97 million US dollars (USD), while the median is SAR 0.62 million (USD0.17 million) and the total cost for the 48 patients is SAR 172.95 million (USD46.12 million). Based on many units leaders' suggestions that transfer to a long-term care facility could alleviate the LSP problem in the ICUs, our team calculated the potential cost savings based on transfer to SDUs after a 60-day PICU stay.

If the alternate policy that our team proposed is implemented; whereby a patient's stay at an ICU is limited to 60 days, after which they will be transferred to an SDU, the mean and median cost per patient would be SAR 2.28 million (USDD 0.60 million) and SAR 0.50 million (USD0.13 million), respectively. The total cost of hospitalization for the 48 patients if the new policy is implemented would be SAR 109.47 million (USDD 29.19 million). The median cost per patient for the current and new policies and their lower and upper quartiles are shown in Figure 4 . The expected mean and median cost savings per patient are SAR 1.32 million (USD0.35 million) and SAR 0.11 million (USDD 0.03 million), respectively. While the potential total cost savings for the 48-patient cohort if the suggested policy were in place at the time of the patients' admission is SAR 63.48 million (USD16.93 million), as shown in Table 1.

\section{Discussion}

This study aimed to highlight and compare the status of LSPs in PICUs and SCICUs in Saudi Arabia and understand the factors that facilitate and hinder the management of the length of time that LSPs spend in ICUs from the perspective of unit leaders from 10 major tertiary hospitals in Riyadh, Saudi Arabia. The issue of LSPs is attributed to many factors related to institutional policies, availability of palliative care resources, family cooperation, and patient 
Table I The 2014 Cost of Hospitalization for Long-Stay Patients in Pediatric Intensive Care Units and the New Proposed Hospitalization Policy Cost

\begin{tabular}{|l|l|l|l|}
\hline \multirow{2}{*}{ Category } & \multicolumn{2}{|c|}{ Cost per Patient in Saudi Riyals (SAR) } & \multirow{2}{*}{ Total Cost in SAR } \\
\cline { 2 - 4 } & \multicolumn{1}{|c|}{ Mean I SD } & \multicolumn{1}{|c|}{ Median (Q1-Q3) } \\
\hline Current policy & $3,603,254.98 \pm 6,396,408.20$ & $623,833.57(232,699.50-301,3174.29)$ & $172,956,239.24$ \\
New proposed policy & $2,280,698.94 \pm 3,850,960.96$ & $500,833.57(218,815.71-1,936,924.29)$ & $109,473,549.23$ \\
Difference & $1,322,556.04 \pm 2,546,165.98$ & $109,675(0.00-1,076,250)$ & $63,482,690.00$ \\
\hline
\end{tabular}

characteristics. ${ }^{1}$ Previous qualitative studies on ICU patients focused on patients and their families' experiences in ICUs, and little was done to integrate health professionals' voices in improving the service to manage the number of LSPs. ${ }^{11}$ The LSPs in the PICUs is reported from several countries with variable impact on the healthcare systems and utilization of resources. ${ }^{12}$

The other aim was to calculate the costs of these LSPs and estimate possible cost savings if such LSPs were transferred to long-term care after a 60-day PICU stay.

In various instances, unit leaders highlighted related institutional factors that contributed to increasing LSPs. Promptly vacating PICU beds is crucial to delaying the transfer of other critically ill patients to the ICU and associated with increased morbidity and mortality. ${ }^{13}$ This study revealed that the process of transferring patients back to the original center of care was described as slow and that a system to transfer them to other facilities was lacking. This was attributed to a lack of a policy to transfer patients back, but the possible adverse events that follow the process of transporting critical cases could be a determining factor. ${ }^{14}$ However, a more recent study demonstrated that following patient transport guidelines is safe even for critically ill patients. ${ }^{15}$

Participants indicated that following a clear policy to accept and transfer patients, as required by national standards, was a facilitating factor in reducing LSPs. In 2013, a Saudi Royal decree mandated the national accreditation of all healthcare institutes by the CBAHI as a prerequisite for renewing their operating license. ${ }^{16}$ According to participants, this initiative played a substantial role in minimizing the number of LSPs. Therefore, setting explicit criteria for accepting patients to the PICU, with a clear refrain from accepting advanced cases that probably will not benefit from aggressive critical care, and developing a policy for care transfer, is crucial. The latter was discussed in previous studies in which developing intermediate care as an alternative to saving costs without jeopardizing the quality of care. ${ }^{17-19}$ This goes along with following the national hospital standards that require an effective policy downgrading the level of care. Nonetheless, participants explained that exercising negative power dynamics among the healthcare system by pushing for the acceptance of cases that would usually not be accepted, and the slow progress of transferring patients might backfire.

The issue of DNR was expected to be raised by participants in the current study, as DNR policies are based on the related Fatwa, the national Code of Ethics Healthcare Practitioners by the Saudi Commission for Health Specialties, and the National Policy and Procedure For DNR Status by the Saudi Health Council. ${ }^{20}$ The decision to transition from intervention to end-of-life care is not without challenges. In addition to the emotional tensions, healthcare professionals must handle intra- and inter-disciplinary tensions. ${ }^{21,22}$ Similarly, this study found that healthcare professionals tend to hold a negative attitude toward actions that lead to downgrading patients' care, especially after building a relationship with the families of LSPs. ${ }^{22}$ The current study proposes that families might compound this unfavorable attitude by refusing to sign DNR approval forms, making complaints to higher authorities, or sharing their perceptions through the media. However, all these actions might compound the negative attitude of healthcare professionals and hinder their effective use of national DNR policies. A national DNR policy was developed and became effective in $2017,{ }^{23}$ which was a positive factor in reducing LSPs. Before that, a considerable degree of heterogeneity in implementing DNRs was observed due to the absence of clear local policies and guidelines. ${ }^{24}$ Compared to the previous pathway of DNR decisions, a main addition of the recently effective policy is family consent regarding DNR implementation. Participants expressed that family consent to DNRs, which minimizes LSPs, was encouraged after the provision of diagnostic tests that justified putting the patient on DNR status. 


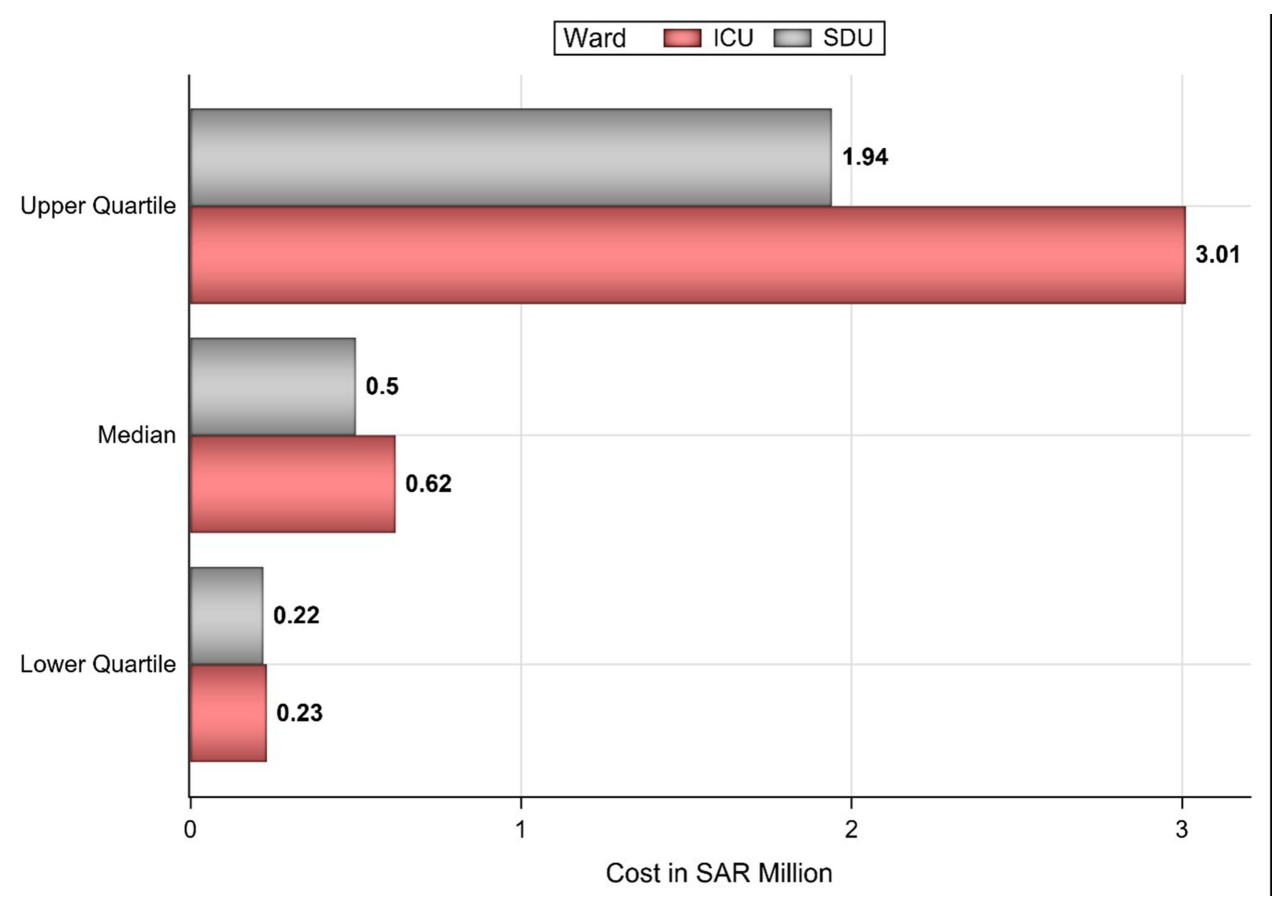

Figure 4 The median cost of hospitalizations for pediatric long-stay patients.

Patient characteristics and case complexity play an essential role in predicting LSPs; ${ }^{25,26}$ however, the current study suggested that having a regular multidisciplinary meeting to discuss PICU patients' approaches is fundamental in reducing LSPs. This evidence is aligned with their suggestion as it shows that a multidisciplinary team approach is beneficial in managing ICU patients, ${ }^{27,28}$ and might, therefore, reduce patients' LOS.

LSPs have poorer prognoses and consume healthcare resources in excess of their numeric proportion compared to short-stay patients. ${ }^{5,26}$ The current study demonstrates that unit leaders believe that available hospital resources should be reserved for short-stay patients, while LSPs should be transferred to palliative care centers. LSPs in PICUs could be addressed to make more critical care beds available during medical emergencies. One proposal during the coronavirus disease 2019 (COVID-19) crisis was to add PICU beds as "surge capacity" to care for critically ill adults. ${ }^{29}$ Globally, this would be particularly important issue during the pandemic, as more ICU beds are urgently needed for the surge to maximize the quality of health care during COVID-19 and future disasters. ${ }^{30}$ Furthermore, as children with COVID-19 who required ICU care had associated comorbidities that could also complicate the issue of LSPs in the PICU setting. ${ }^{31}$

We suggest a model of transferring LSPs to SDUs after 60 days to provide financial savings and free up more critical care beds. Several other models were also reported that saved the expense of LSPs in ICUs. Snell et al described their hospital's implementation of an ICU survivor clinic and compared it to the post-discharge usual care. $^{32}$ They found decreased mortality and net annual cost savings that ranged from USD247,052 to USD424,846 during the 13 months that were analyzed. Another adult ICU model dedicated weaning center for tracheostomized difficult-to-wean patients admitted to respiratory intermediate care units. It resulted in overall $39,845 \pm 22,578$ euros mean cost savings per patient compared to ICU. ${ }^{33}$ The utilization of long-term medical home care is another possible solution for LSPs. Mosquera et al reported that enhanced medical home care prevented serious illness and PICU admissions and promoted cost reductions for children with chronic illnesses. ${ }^{34}$ This would be in line with one of the aims of the new Vision 2030 National Transformation Program for healthcare in 
promoting home healthcare services. ${ }^{35}$ Additionally, more savings are expected if the study participants' suggestions to reduce LSPs are addressed.

\section{Study Limitations}

This is the first study to explore issues to mitigate the LSPs' burden from healthcare leaders' perspective. We acknowledge that this study was based on the findings of interview the units' leader, with the intention of exploring the reality, so we can further develop explanatory models and theories. ${ }^{36}$ Additionally, the study only included ICUs leaders from most tertiary care hospitals in the city of Riyadh, which limits the generalizability of the findings. That said, due to the qualitative methodology used, the reader should think of the context before transferring this study's results to other settings. Furthermore, the researchers feel that in-person interviews would have elicited richer responses. The choice of online and voice messaging was to accommodate the participants' largely variable and tight schedules and ensure their valuable response on the matter.

Regarding the cost-savings of the proposed model of transfer to a long-term care facility after 60 days, this was suggested by the authors based on experts' opinion; therefore, the optimal timing needs to be sought through future research. The hospitals that we included were governmental hospitals that are primarily not insurance-based. The healthcare system in Saudi Arabia is undergoing transformation towards restructuring their healthcare system to privatize public hospitals and introduce a variety of healthcare reforms that may use a mixture of different payment models including insurance coverage, ${ }^{37,38}$ so future research potentials are abundant, especially that such changes present an interesting and insightful case for the challenges in fundamentally changing a country's healthcare system. ${ }^{37}$

\section{Conclusions}

Identifying the challenges that contributed to LSPs, constructed on ICU leaders' perspective, and implementing strategies to reduce patients' LOS in ICUs is crucial to informing systems and policies to lower costs and improve the utilization of ICU resources. Creating explicit guidelines to govern acceptance and transfer of critically ill patients, providing multidisciplinary communication, educating the public and healthcare workers about the dimensions of DNR status, and investing in transferring LSPs to SDUs are the measures that were proposed by healthcare workers to reduce LSPs.

\section{Abbreviations}

DNR, do-not-resuscitate; LOS, length of stay; LSP, longstay patients; PICUs, pediatric intensive care units; SCICUs, pediatric surgical cardiac intensive care units.

\section{Data Sharing Statement}

All the data for this study will be made available upon reasonable request.

\section{Ethics Approval and Consent to Participate}

The study was approved by the institutional review board at King Saud University (approval \#13/3793/IRB). All participants gave consent to have their data published, and this was electronically included in the coversheet of the electronic survey that "completing the survey includes the participant's consent to participate in this IRB-Approved research and to publish the results." This study was conducted in accordance with the Declaration of Helsinki.

\section{Consent for Publication}

All authors gave their consent for publication.

\section{Acknowledgments}

The authors wish to acknowledge the efforts and contributions of Dr. Saleh Alshehri, Dr. Abdulrahman Alshehri, Dr. Yasser Alheraish, Dr. Hanan Abuhassan, Dr. Taerq Alanezi, Alanazi K, Alfryian T, Alghamdi A, and Almudi M, and all the participating PICU/SCICU leaders for their valuable contributions. The authors are grateful to the Deanship of Scientific Research, King Saud University for funding through Vice Deanship of Scientific Research Chairs. We would also like to thank Research chair for Evidence Based Health Care and Knowledge Translation for support and Dr. Samia Ahmed Ismaeil for the effort.

\section{Author Contributions}

All authors made a significant contribution to the work reported, whether that is in the conception, study design, execution, acquisition of data, analysis and interpretation, or in all these areas; took part in drafting, revising or critically reviewing the article; gave final approval of the version to be published; have agreed on the journal to which the article has been submitted; and agreed to be accountable for all aspects of the work. 


\section{Disclosure}

The authors reported no conflicts of interest for this work.

An abstract has been presented in the Society of Critical Care Medicine and the World Federation of Pediatric Intensive and Critical Care Societies Conference in 2020 and it is published in their journal: https://journals.lww. com/pccmjournal/Fulltext/2021/03001/P0317_ 1844 PREDICTORS_AND_DIRECT_COST.375.aspx.

\section{References}

1. Pollack MM, Wilkinson JD, Glass NL. Long-stay pediatric intensive care unit patients: outcome and resource utilization. Pediatrics. 1987;80(6):855-860.

2. Conlon NP, Breatnach C, O'Hare BP, Mannion DW, Lyons BJ. Health-related quality of life after prolonged pediatric intensive care unit stay. Pediatr Crit Care Med. 2009;10(1):41-44. doi:10.1097/ PCC.0b013e31819371f6

3. van der Heide P, Hassing MB, Gemke RJ. Characteristics and outcome of long-stay patients in a paediatric intensive care unit: a case-control study. Acta Paediatr. 2004;93(8):1070-1074. doi:10.1111/j.1651-2227.2004.tb02720.x

4. Naghib S, van der Starre C, Gischler SJ, Joosten KF, Tibboel D. Mortality in very long-stay pediatric intensive care unit patients and incidence of withdrawal of treatment. Intensive Care Med. 2010;36 (1):131-136. doi:10.1007/s00134-009-1693-z

5. Gonzalez-Cortes R, Lopez-Herce-Cid J, Garcia-Figueruelo A, Tesorero-Carcedo G, Botran-Prieto M, Carrillo-Alvarez A. [Prolonged stay in pediatric intensive care units: mortality and healthcare resource consumption]. Med Intensiva. 2011;35(7):417-423. doi:10.1016/j.medin.2011.04.004.

6. Namachivayam P, Taylor A, Montague T, et al. Long-stay children in intensive care: long-term functional outcome and quality of life from a 20-yr institutional study. Pediatr Crit Care Med. 2012;13 (5):520-528. doi:10.1097/PCC.0b013e31824fb989

7. Shapiro MC, Henderson CM, Hutton N, Boss RD. Defining pediatric chronic critical illness for clinical care, research, and policy. Hospital Pediatr. 2017;7(4):236-244. doi:10.1542/hpeds.2016-0107

8. World Bank staff estimates based on age/sex distributions of united nations population division's world population prospects: 2017 Revision; 2017.

9. Temsah MA, Al-Eyadhy AA, Al-Sohime FM, et al. Long-stay patients in pediatric intensive care units. Five-years, 2-points, cross-sectional study. Saudi Med J. 2020;41(11):1187-1196. doi:10.15537/smj.2020.11.25450

10. Elo $\mathrm{S}$, Kyngas $\mathrm{H}$. The qualitative content analysis process. $J A d v$ Nurs. 2008;62(1):107-115. doi:10.1111/j.1365-2648.2007.04569.x

11. Geoghegan S, Oulton K, Bull C, Brierley J, Peters M, Wray J. The experience of long-stay parents in the ICU: a qualitative study of parent and staff perspectives. Pediatr Crit Care Med. 2016;17(11): e496-e501. doi:10.1097/pcc.0000000000000949

12. Briassoulis G, Filippou O, Natsi L, Mavrikiou M, Hatzis T. Acute and chronic paediatric intensive care patients: current trends and perspectives on resource utilization. Qjm. 2004;97(8):507-518. doi:10.1093/qjmed/hch087

13. Young MP, Gooder VJ, McBride K, James B, Fisher ES. Inpatient transfers to the intensive care unit: delays are associated with increased mortality and morbidity. J Gen Intern Med. 2003;18 (2):77-83. doi:10.1046/j.1525-1497.2003.20441.x

14. Barry PW, Ralston C. Adverse events occurring during interhospital transfer of the critically ill. Arch Dis Child. 1994;71(1):8-11. doi:10.1136/adc.71.1.8
15. Uusaro A, Parviainen I, Takala J, Ruokonen E. Safe long-distance interhospital ground transfer of critically ill patients with acute severe unstable respiratory and circulatory failure. Intensive Care Med. 2002;28(8):1122-1125. doi:10.1007/s00134-002-1348-9

16. CBAHI At A Glance; 2021. Available from: https://portal.cbahi.gov. sa/english/about-us/cbahi-at-a-glance. Accessed January 9, 2021.

17. Zimmerman JE, Wagner DP, Knaus WA, Williams JF, Kolakowski D, Draper EA. The use of risk predictions to identify candidates for intermediate care units. Implications for intensive care utilization and cost. Chest. 1995;108(2):490-499. doi:10.1378/chest.108.2.490

18. Zimmerman JE, Wagner DP, Sun X, Knaus WA, Draper EA. Planning patient services for intermediate care units: insights based on care for intensive care unit low-risk monitor admissions. Crit Care Med. 1996;24(10):1626-1632. doi:10.1097/00003246-19961000000005

19. Mounsey JP, Griffith MJ, Heaviside DW, Brown AH, Reid DS. Determinants of the length of stay in intensive care and in hospital after coronary artery surgery. $B r$ Heart $J$. 1995;73(1):92-98. doi:10.1136/hrt.73.1.92

20. Arabi Y, Al-Sayyari A, Al Moamary M. Shifting paradigm: from "No Code" and "Do-Not-Resuscitate" to "Goals of Care" policies. Commentary. Ann Thorac Med. 2018;13(2):67-71. doi:10.4103/atm. ATM 39317

21. Coombs MA, Addington-Hall J, Long-Sutehall T. Challenges in transition from intervention to end of life care in intensive care: a qualitative study. Int $J$ Nurs Stud. 2012;49(5):519-527. doi:10.1016/j.ijnurstu.2011.10.019

22. Wilson ME, Rhudy LM, Ballinger BA, Tescher AN, Pickering BW, Gajic O. Factors that contribute to physician variability in decisions to limit life support in the ICU: a qualitative study. Intensive Care Med. 2013;39(6):1009-1018. doi:10.1007/s00134-013-2896-x

23. Council SH. National policy and procedure for do-not-resuscitate (DNR) Status. Rabi II. 2017;1438.

24. Amoudi AS, Albar MH, Bokhari AM, Yahya SH, Merdad AA. Perspectives of interns and residents toward do-not-resuscitate policies in Saudi Arabia. Adv Med Educ Pract. 2016;7:165-170. doi:10.2147/amep.s99441

25. Brown KL, Ridout DA, Goldman AP, Hoskote A, Penny DJ. Risk factors for long intensive care unit stay after cardiopulmonary bypass in children. Crit Care Med. 2003;31(1):28-33. doi:10.1097/ 00003246-200301000-00004

26. Marcin JP, Slonim AD, Pollack MM, Ruttimann UE. Long-stay patients in the pediatric intensive care unit. Crit Care Med. 2001;29 (3):652-657. doi:10.1097/00003246-200103000-00035

27. Kocyildirim E, Kanani M, Roebuck D, et al. Long-segment tracheal stenosis: slide tracheoplasty and a multidisciplinary approach improve outcomes and reduce costs. J Thorac Cardiovasc Surg. 2004;128(6):876-882. doi:10.1016/j.jtcvs.2004.07.008

28. Dutton RP, Cooper C, Jones A, Leone S, Kramer ME, Scalea TM. Daily multidisciplinary rounds shorten length of stay for trauma patients. $J$ Trauma. 2003;55(5):913-919. doi:10.1097/01. ta.0000093395.34097.56

29. Remy KE, Verhoef PA, Malone JR, et al. Caring for Critically Ill Adults With Coronavirus Disease 2019 in a PICU: recommendations by Dual Trained Intensivists. Pediatr Crit Care Med. 2020;21 (7):607-619. doi:10.1097/pcc.0000000000002429

30. Sen-Crowe B, Sutherland M, McKenney M, Elkbuli A, Closer Look A. Into global hospital beds capacity and resource shortages during the COVID-19 Pandemic. J Surg Res. 2021;260:56-63. doi:10.1016/j.jss.2020.11.062

31. Alsohime F, Temsah MH, Al-Nemri AM, Somily AM, Al-Subaie S. COVID-19 infection prevalence in pediatric population: etiology, clinical presentation, and outcome. J Infect Public Health. 2020;13 (12):1791-1796. doi:10.1016/j.jiph.2020.10.008 
32. Snell KP, Beiter CL, Hall EL, et al. A Novel Approach to ICU survivor care: a population health quality improvement project. Crit Care Med. 2020;48(12):e1164-e1170. doi:10.1097/ ccm.0000000000004579

33. Carpenè N, Vagheggini G, Panait E, Gabbrielli L, Ambrosino N. A proposal of a new model for long-term weaning: respiratory intensive care unit and weaning center. Respir Med. 2010;104 (10):1505-1511. doi:10.1016/j.rmed.2010.05.012

34. Mosquera RA, Avritscher EBC, Samuels CL, et al. Effect of an enhanced medical home on serious illness and cost of care among high-risk children with chronic illness: a randomized clinical trial. JAMA. 2014;312(24):2640-2648. doi:10.1001/jama.2014.16419
35. National Transformation Program; 2021 Available from: https:// vision2030.gov.sa/sites/default/files/attachments/NTP\%20English\% 20Public\%20Document_2810.pdf. Accessed January 4, 2021.

36. Morse JM, Field PA. The purpose of qualitative research. 1996. Nurs Res. 1-17. Springer.

37. Walston S, Al-Harbi Y, Al-Omar B. The changing face of healthcare in Saudi Arabia. Ann Saudi Med. 2008;28(4):243-250. doi:10.5144/ 0256-4947.2008.243

38. AlRuthia Y, Abdulaziz Bin Aydan N, Sulaiman Alorf N, Asiri Y. How can Saudi Arabia reform its public hospital payment models? A narrative review. Saudi Pharm J. 2020;28(12):1520-1525. doi:10.1016/j.jsps.2020.09.020

\section{Publish your work in this journal}

Risk Management and Healthcare Policy is an international, peerreviewed, open access journal focusing on all aspects of public health, policy, and preventative measures to promote good health and improve morbidity and mortality in the population. The journal welcomes submitted papers covering original research, basic science, clinical \& epidemiological studies, reviews and evaluations, guidelines, expert opinion and commentary, case reports and extended reports. The manuscript management system is completely online and includes a very quick and fair peer-review system, which is all easy to use. Visit http://www.dovepress.com/testimonials.php to read real quotes from published authors. 